\title{
recillunds
}

Revista Científica Mundo de la Investigación y el Conocimiento

\author{
Wendy Lorena Ocampo Ulloa ${ }^{\mathrm{a}}$; Nora Hursula Huilcapi Masacon ${ }^{\mathrm{b}}$; Andrés \\ Fernando Cifuentes Rodríguez ${ }^{\mathrm{c}}$
}

La estructura organizacional en función del comportamiento del capital humano en

las organizaciones

The organizational structure based on the behavior of human capital in

organizations

Revista Científica Mundo de la Investigación y el Conocimiento. Vol. 3 núm. 4., diciembre, ISSN: 2588-073X, 2019, pp. 114-137

DOI: $10.26820 /$ recimundo/3.(4).diciembre.2019.114-137

URL: http://recimundo.com/index.php/es/article/view/655

Código UNESCO: 5311 Organización y Dirección de Empresas

Tipo de Investigación: Artículo de Revisión

(C) RECIMUNDO; Editorial Saberes del Conocimiento, 2019

Recibido: 15/09/2019

Aceptado: 23/11/2019

Publicado: 30/12/2019

Correspondencia: director@recimundo.com

a. Magister en Administración de Empresas; Ingeniera en Empresas y Administración de Negocios; Técnica Ejecutiva en Negocios; Tecnóloga Administradora de Empresas; Docente de la Universidad Técnica de Babahoyo; Facultad de Administración Finanzas e Informática; Babahoyo, Ecuador; wocampo@utb.edu.ec

b. Magister Ejecutivo en Dirección de Empresas con Énfasis en Gerencia Estratégica; Especialista en Gerencia de Proyectos; Diploma Superior en Investigación de la Educación a Distancia; Diplomado Superior en Gerencia de Marketing; Diplomado en Gerencia de Marketing; Licenciada en Ciencias de la Educación en la Especialización Comercio y Administración; Profesora de Segunda Enseñanza en la Especialización de Comercio y Administración; Docente de la Universidad Técnica de Babahoyo; Facultad de Administración Finanzas e Informática; Babahoyo, Ecuador; nuhulcapi@utb.edu.ec

c. Master Universitario en Gestión Internacional del Turismo; Ingeniero en Planificación Turística énfasis Operación Turística; Docente de la Universidad Técnica de Babahoyo; Facultad de Administración Finanzas e Informática; Babahoyo, Ecuador; acifuentes@utb.edu.ec 


\section{La estructura organizacional en función del comportamiento del capital humano en las organizaciones}

Vol. 3, núm. 4., (2019)

Wendy Lorena Ocampo Ulloa; Nora Hursula Huilcapi Masacon; Andrés Fernando Cifuentes

Rodríguez

\section{RESUMEN}

Este estudio va enfocado en el comportamiento del talento humano en función a una estructura funcional sea esta horizontal y vertical, teniendo en consideración que una estructura funcional horizontal es aquella que a los empleados se les otorga la misma capacidad de tomar decisiones, sin tener la autorización de un superior; en cambio la estructura funcional vertical es aquella que se distribuye las funciones en base a un nivel jerárquico, para lo cual se ha desarrollado un estudio que permita analizar si el comportamiento de los colaboradores dentro de una organización depende de su estructura organizacional, y cómo influye el mismo en las actividades que se realicen.

El presente artículo tiene como objetivo principal exponer una perspectiva general referente a la conducta que tiene el talento humano en base a la estructura vertical y horizontal de una organización, para ello es necesario tomar en consideración que el personal es el activo más importante dentro de toda entidad, pues es quien apoya a que la misma pueda lograr sus objetivos y metas mediante el empleo y coordinación de esfuerzos para lograr el éxito necesario y por ende se necesita de una estructura organizativa que permita administrar de manera adecuada los recursos que se poseen.

Por consiguiente cabe recalcar que para lograr este objetivo es necesario emplear procesos experimentales o materiales y métodos, tales como una investigación exhaustiva y exploratoria, así como métodos estadísticos y matemáticos para así determinar cuál es el proceder del talento humano en la estructura organizacional. Los diferentes procesos que inciden en el comportamiento del personal y que acontecen sus diferentes niveles de análisis permiten establecer adecuadas estrategias para comprender y mejorar los retos del cambio empresarial, convirtiéndose así en la llave maestra para alcanzar un liderazgo eficaz.

Palabras Claves: Capital Humano; Comportamiento; Organización; Recursos; Administrar; Liderazgo. 


\section{La estructura organizacional en función del comportamiento del capital humano en las organizaciones}

Vol. 3, núm. 4., (2019)

Wendy Lorena Ocampo Ulloa; Nora Hursula Huilcapi Masacon; Andrés Fernando Cifuentes Rodríguez

\section{ABSTRACT}

This study focuses on the behavior of human talent according to a functional structure, whether horizontal or vertical, taking into account that a horizontal functional structure is one that gives employees the same ability to make decisions without having the authorization of a superior; instead the vertical functional structure is one that distributes the functions based on a hierarchical level, for which a study has been developed to analyze whether the behavior of collaborators within an organization depends on its organizational structure, and how it influences the same in the activities that are carried out.

The main objective of this article is to present a general perspective regarding the behavior of human talent based on the vertical and horizontal structure of an organization, for this it is necessary to take into consideration that personnel is the most important asset in all entity, because it is who supports that it can achieve its objectives and goals through the use and coordination of efforts to achieve the necessary success and therefore an organizational structure is needed to manage properly the resources that are possessed.

Therefore, it should be emphasized that in order to achieve this objective it is necessary to use experimental or material processes and methods, such as exhaustive and exploratory research, as well as statistical and mathematical methods, in order to determine the human talent's behavior in the organizational structure. The different processes that affect the behavior of the staff and that occur at different levels of analysis allow to establish adequate strategies to understand and improve the challenges of business change, thus becoming the master key to achieve effective leadership.

Keywords: Human Talent; Behavior; Organization; Resources; Manage; Leadership. 


\section{La estructura organizacional en función del comportamiento del capital humano en las organizaciones}

Vol. 3, núm. 4., (2019)

Wendy Lorena Ocampo Ulloa; Nora Hursula Huilcapi Masacon; Andrés Fernando Cifuentes Rodríguez

\section{Introducción.}

En la actualidad la manera en que se gestiona y administra las diferentes organizaciones existentes han tenido ciertos cambios debido a distintos factores ya sea económicos o sociales que son producto de un mundo globalizado, por ende para que estas se adapten a las nuevas propensiones de mercado es necesario que las mismas dejen atrás las estructuras rígidas y empleen sistemas organizacionales que sean más flexibles para así facilitar que el talento humano tenga claro los objetivos de la empresa y de paso a que su organización sea competitiva y eficiente.

Así mismo la conducta del Personal en las Organizaciones ha generado un gran énfasis en el desarrollo de la productividad de las mismas, pues con el adecuado manejo de los recursos ha permitido que la estructura de las organizaciones les ayude a llevar una buena administración.

De la misma manera es necesario denotar que la estructura organizacional vertical hoy en día es la que se aplica en la mayoría de las empresas, donde está basada en un nivel jerárquico, en la que cuenta con gerentes, jefes departamentales y personal de servicios. Dentro de la cual cada departamento tiene bien definido cuales son las responsabilidades que debe de cumplir, el sistema vertical ha provocado que el personal actué y cumpla de manera adecuada sus labores, de tal manera que diariamente están siendo controlados con el fin de que cumplan sus labores.

Por otro lado la estructura organizacional horizontal se utiliza con menos frecuencia en las empresas, pues se encarga de que el personal aplique sus propias capacidades para tomar decisiones, dentro del cual en estas organizaciones existe la libertad de dar opiniones, ideas, 


\section{La estructura organizacional en función del comportamiento del capital humano en las organizaciones}

Vol. 3, núm. 4., (2019)

Wendy Lorena Ocampo Ulloa; Nora Hursula Huilcapi Masacon; Andrés Fernando Cifuentes Rodríguez

proyectos, sin temor a alguno de demostrar sus habilidades por discriminación o exclusión por la actividad que realice dentro de ellas. A veces esa libertad puede ocasionar acciones perjudiciales para las empresas por el inadecuado sentido de apoyo.

\section{Desarrollo.}

En este mundo versátil e influenciado en todos los ámbitos por la globalización y la tecnología, las organizaciones como sistemas abiertos, en la misión y visión reflejan como designios crear y mantener políticas, dirigidas al logro de un ambiente favorable, en donde el personal se sienta motivado a realizar funciones que contribuyan a lograr las metas y objetivos preestablecidos mediante la utilización racional de sus recursos.

Por consiguiente es necesario denotar que los gerentes son los responsables de bosquejar la estructura que se empleará dentro de la organización, debido a que mediante la misma se va a determinar que tareas deberán hacerse, quien las debe de ejecutar, como se llevará a cabo la división del trabajo y donde se toman las decisiones. En este sentido cabe recalcar que la estructura que se diseñe en la empresa tiene una mayor influencia en el comportamiento de los individuos que desempeñan sus labores en la misma.

\section{La Organización}

Según (Martínez, Tipos de organización y estructura organizacional, 2014) "La organización se refiere al establecimiento y agrupación de actividades y recursos necesarios que interactúen entre sí a través de una coordinación horizontal y vertical para el cumplimiento de los objetivos contemplados en la estructura de la empresa". 


\section{La estructura organizacional en función del comportamiento del capital humano en las organizaciones}

Vol. 3, núm. 4., (2019)

Wendy Lorena Ocampo Ulloa; Nora Hursula Huilcapi Masacon; Andrés Fernando Cifuentes Rodríguez

De modo general la organización hace referencia a un conjunto de estructuras que dependen de cómo se desenvuelve el talento humano y del ambiente en el que se encuentra para llegar a ser totalmente exitosa. En donde es necesario que exista una ardua administración en los recursos que se posee para así contribuir al logro de los estándares descritos en la definición de funciones de la entidad.

Importancia del talento humano en las organizaciones

Es importante que la gestión humana sea medida a través de indicadores en cada uno de sus subsistemas y también en aquellos aspectos de relevancia para la organización como, por ejemplo: Capacitación; siniestralidad laboral; rotación de personal; ausentismo, entre otros. Por lo tanto, los indicadores de la gestión humana (IGH), se miden en una constante de tiempo, que permite realizar comparaciones mes a mes y año a año, o un historial de los últimos cinco años, de empresa en empresa, incluso de país en país. (Armas Ortega, LLanos Enacalada, \& Traverso Holguin , 2017, pág. 64)

El talento humano forma parte esencial para que la organización pueda figurarse con una notable ventaja frente a la competencia. En efecto se debe de prestar mayor atención a como ellos reaccionan ante las estructuras que las entidades implementan y es necesario que dichas dimensiones sean totalmente flexibles, es decir se adapten a cualquier cambio con facilidad.

"En toda organización empresarial el talento humano y la calidad del mismo es el recurso fundamental, ya que dé él depende el éxito de la entidad” (Mora, 2014, pág. 4) 


\section{La estructura organizacional en función del comportamiento del capital humano en las organizaciones}

Vol. 3, núm. 4., (2019)

Wendy Lorena Ocampo Ulloa; Nora Hursula Huilcapi Masacon; Andrés Fernando Cifuentes Rodríguez

El talento humano es esencial, ya que el mismo efectúa un adecuado manejo de los diferentes recursos que posee la empresa. Los individuos que pertenecen a la organización son los encargados de plantear y producir los bienes y servicios, además de establecer los capitales económicos, y de implantar las metas y tácticas corporativas.

El innovar con frecuencia permite que la organización se mantenga con vida y más allá de ello, siempre captará la atención del usuario. El desarrollo de la cultura es de vital importancia para los individuos ya que de esta manera ayuda a definir fronteras, establecer diferencias entre una organización y otras, conllevando a que exista un sentimiento de identidad entre ellas. (Pazmiño Romero, Caicedo Monserrate, \& Ocampo Ulloa, 2018, pág. 15)

Para mantener un constante crecimiento en las organizaciones no solo es necesario que el talento humano se desenvuelva con eficiencia, sino también es esencial que el mismo mediante el empleo de distintas técnicas efectué el desarrollo de nuevos productos o servicios, visto que así se dará paso a que la entidad tenga una larga duración en el entorno en el que se desenvuelve.

\section{¿Qué es una estructura organizacional?}

Una estructura organizacional define la manera en que las actividades del puesto de trabajo se dividen, agrupan y coordinan. Los gerentes tienen que considerar seis elementos fundamentales cuando diseñan la estructura de su organización: especialización en el trabajo, departamentalización, cadena de mando, extensión de control, centralización y descentralización y formalización. (Stephen \& Judge, 2013, pág. 480) 


\section{La estructura organizacional en función del comportamiento del capital humano en las organizaciones}

Vol. 3, núm. 4., (2019)

Wendy Lorena Ocampo Ulloa; Nora Hursula Huilcapi Masacon; Andrés Fernando Cifuentes Rodríguez

De la igual manera uno de los elementos primordiales dentro de las distintas organizaciones son las personas, ya que estas interactúan de carácter positivo al realizar las funciones esenciales de la empresa para así contribuir al logro de las metas planeadas. Es por ello que se hace necesario que los gerentes estructuren y sistematicen de forma clara los recursos que posee la organización con el fin de que se haga posible conseguir los propósitos desarrollados. En efecto es necesario emplear una estructura organizacional que se adapte a las actividades que desempeña el talento humano.

La estructura organizacional forma una parte fundamental dentro de las diferentes empresas, puesto que permite que se dividan las actividades que desenvuelve el talento humano con el fin de que sean desarrolladas de forma eficaz y eficiente para que así contribuyan al logro de las metas y objetivos empresariales.

Sin embargo aun cuando las responsabilidades de la empresa están delegadas en diferentes secciones, en el día de hoy lo que las entidades buscan es estructurase de manera que exista una mayor coordinación en las actividades laborales y a su vez que estas estructuras sean flexibles y se adapten rápidamente a los cambios que puede provocar el entorno, pues el mismo es muy cambiante ante la sociedad.

Debe diseñarse para determinar quien realizará cuales tareas y quien será responsable de que resultados; para eliminar los obstáculos al desempeño que resulta ante la confusión e incertidumbre respecto a la asignación de actividades y para tender redes en cuanto a la toma de decisiones y comunicación que respondan y sirvan de apoyo a los objetivos empresariales. (Martínez, Tipos de organización y estructura organizacional, 2014) 


\section{La estructura organizacional en función del comportamiento del capital humano en las organizaciones}

Vol. 3, núm. 4., (2019)

Wendy Lorena Ocampo Ulloa; Nora Hursula Huilcapi Masacon; Andrés Fernando Cifuentes Rodríguez

Dentro de la estructura organizacional se pueden mencionar tres componentes claves, mismos que son:

- Mediante la estructura organizacional se distingue el grado de jerarquía del talento humano que constituye la organización, así como el control que deben de ejercer los niveles gerenciales.

- El desarrollo de la estructura en una organización permite delegar las actividades que se deben de ejecutar en los departamentos o áreas correspondientes a la empresa.

- Finalmente, el empleo de una buena estructura organizacional da paso a que exista una excelente comunicación entre los integrantes de los diferentes departamentos de la empresa. Así mismo se permite la integración de los esfuerzos laborales.

Las personas indefectiblemente se encuentran inmersas en relaciones sociales, en convivencia grupal, en sociedad, es razonable que experimenten influencias importantes por parte de algunos integrantes del grupo o, de los procesos grupales. Actualmente, los equipos o grupos de trabajo se han convertido en una pieza central de la estructura organizacional, ya que el trabajo colaborativo y en equipo es cada vez más importante de cara a los constantes desafíos. (Llanos Encalada, Pacheco Rodríguez, Romero Vélez, Coello Arrata, \& Armas Ortega, 2016, pág. 79)

De modo que toda entidad debe de contar con una estructura bien diseñada ya que de esta depende que el talento humano tenga una mayor comprensión de las funciones que debe de desarrollar, es decir se debe de asignar el dominio y las ocupaciones de manera adecuada y de 


\section{La estructura organizacional en función del comportamiento del capital humano en las organizaciones}

Vol. 3, núm. 4., (2019)

Wendy Lorena Ocampo Ulloa; Nora Hursula Huilcapi Masacon; Andrés Fernando Cifuentes Rodríguez

equitativa. En este sentido se establecen dos tipologías de formas organizativas que se basan en la estructura vertical u horizontal.

\section{Estructura vertical}

Con el propósito de que la conducta organizacional sea vigorosa, debe existir una intervención activa de los gerentes debido a que son ellos quienes han de construir los objetivos y optar las vías que permitan alcanzar las metas organizacionales. Los administradores deben de tener la capacidad de establecer sus objetivos en términos de optimizar la producción, mantener la eficacia, lograr la satisfacción y generar una estructura flexible.

Una entidad que adopta una estructura organizativa vertical es aquella en donde se da una distribución del poder bien definido, dicho de otra manera las responsabilidades se efectúan de arriba hacia abajo. Es decir el gerente es aquella persona quien toma las decisiones con respecto a su organización. (Mejias, La estructura organizativa vertical y la horizontal, 2019)

Por lo tanto, la estructura vertical de una determinada organización se basa en una dimensión en donde el poder que se ejerce recae en los niveles directivos, con el fin de que ellos controlen de manera directa las actividades que realiza el talento humano. En este sentido el comportamiento que tiene el personal frente a este tipo de disposición se basa en que deben de acatar las órdenes que son designadas por los altos mando lo que asienta que se desenvuelvan de manera efectiva y contribuyan en sentido eficiente a que se puedan lograr las metas y objetivos que fueron definidos por la entidad durante el proceso de planificación. 


\section{La estructura organizacional en función del comportamiento del capital humano en las organizaciones}

Vol. 3, núm. 4., (2019)

Wendy Lorena Ocampo Ulloa; Nora Hursula Huilcapi Masacon; Andrés Fernando Cifuentes Rodríguez

Así mismo el talento humano que pertenece a niveles más bajos deberá de efectuar sus funciones conforme a las metas dispuestas por los altos directivos y los altos mandos deben de tener conocimiento respecto a las actividades que efectúan su personal.

Este tipo de organización es altamente jerárquica, con niveles de graduación de responsabilidad y poder bien definidas, en la cual cada quien tiene una función y obedece a los elementos superiores. Ejemplos de esta organización son los sistemas educacionales y gubernamentales, ya que están construidos sobre el concepto de que la autoridad proviene de una persona a quienes muchos obedecen. (Mejias, La estructura organizativa vertical y la horizontal, 2019)

\section{Diseño de una estructura vertical}

En este tipo de organigramas, los puestos se representan por cuadros. Las líneas representan la autoridad y el flujo de la comunicación. Los puestos o cuadros situados arriba, tienen autoridad sobre los situados más abajo. Y estos últimos, dependen o están subordinados a los de arriba. Es decir, exista una graduación jerárquica descendente. (Gonzales, 2018)

Dicho de otra manera dentro del diseño de una estructura vertical, los puestos que se han perfilado deben de simbolizase por cuadros, de tal manera que permitan una mejor comprensión de las figuras que ejercen el mando y en quienes recae la obligación de profesar una buena comunicación con los niveles medios y bajos de la entidad. Debido a que en esta tipología de estructura el poder recae principalmente en el gerente, mismo que desarrolla el papel de líder y es quien ejerce la motivación y control para con su personal. 


\section{La estructura organizacional en función del comportamiento del capital humano en las organizaciones}

Vol. 3, núm. 4., (2019)

Wendy Lorena Ocampo Ulloa; Nora Hursula Huilcapi Masacon; Andrés Fernando Cifuentes Rodríguez

Por otro lado el diseño organizacional debe de proporcionar una excelente comunicación entre el talento humano que labora en la entidad con sus jefes departamentales para así lograr una buena ejecución en las tareas de la empresa. Es precisamente por ello que los gerentes crean vínculos de información que permitan realizar una buena comunicación y combinación entre el talento humano y los demás elementos de la organización.

Comportamiento del talento humano en función a la estructura vertical de la organización

Se trata de una estructura vertical en la medida que cada grupo funcional está integrado verticalmente desde la parte inferior hasta la parte superior de la organización. Así, un vicepresidente de Finanzas tiene a su cargo el trabajo de todo el equipo agrupado en el departamento de su especialidad. (Conecionesan, 2017)

Por lo tanto el talento humano cuando labora en base a una estructura vertical tiende a ejecutar sus tareas de acorde a lo que establece la autoridad. Si bien esto proporciona una eficacia en cada grupo de trabajo, pero también podría provocar deterioro en el proceso de distribución de la información, volviéndola cada vez más lenta y rígido. En este sentido también es necesario considerar que la mayoría de las organizaciones aplican la estructura vertical debido a que la misma le permite al gerente ejercer un gran dominio sobre sus empleados, es decir la comunicación se vuelve tardía debido al exceso de autoridad las entidades.

Las distintas entidades que aplican esta dimensión suelen ser más eficientes porque siempre existe un nivel autoritario que está controlado de manera constante a su personal y además es quien toma las decisiones. 


\section{La estructura organizacional en función del comportamiento del capital humano en las organizaciones}

Vol. 3, núm. 4., (2019)

Wendy Lorena Ocampo Ulloa; Nora Hursula Huilcapi Masacon; Andrés Fernando Cifuentes Rodríguez

\section{Estructura horizontal}

Con el fin de coordinar las actividades de una organización de una manera eficaz, la gestión de la misma pone en práctica a las estructuras que mejor se adapten a esta. Estas estructuras determinan cómo las diferentes personas al interior de la organización se relacionan entre sí. En la estructura organizativa funcional, empleados con habilidades similares trabajan en el mismo departamento bajo la supervisión de una persona con un alto nivel de habilidad. En la estructura organizativa del producto, la organización está dividida de acuerdo a los productos ofrecidos o fabricados. Las estructuras de matriz organizacional adoptan estructuras organizativas funcionales y de producto. (Logan, 2018)

Así mismo se debe de resaltar la importancia y relevancia que posee el desarrollo de una estructura organizacional, debido a que esta es aquella que complementa que cada empleado conozca cuáles son sus atribuciones y deberes que debe de cumplir a cabalidad y con un excelente énfasis.

De manera análoga una estructura organizativa horizontal (estructura plana) es aquella en la que se han eliminado la mayoría de los niveles de mandos medios y de sus funciones, con lo que la alta dirección está en el contacto directo con los trabajadores de primera línea (vendedores, servicio técnico, etc.), los empleados de la planta de producción y clientes (Enciclipedia Financiera, 2014).

Es decir que la estructura horizontal es aquella en la que los altos mandos de la organización ya no ejercen su rol de poder dentro de la organización, sino más bien en esta 


\section{La estructura organizacional en función del comportamiento del capital humano en las organizaciones}

Vol. 3, núm. 4., (2019)

Wendy Lorena Ocampo Ulloa; Nora Hursula Huilcapi Masacon; Andrés Fernando Cifuentes Rodríguez

tipología de dimensión se capacita constantemente al talento humano. Es allí en donde se direcciona que el comportamiento del talento humano en concordancia con la estructura horizontal se da en base a que este apoya en la toma de decisiones importantes para la compañía. Además el talento humano es parte esencial para lograr el éxito de la compañía.

Una de las principales ventajas que tiene la utilización de la estructura horizontal se basa en que permite que las organizaciones pueden diferenciarse entre otras, debido a que pueden responder con rapidez a los cambios que genera el entorno, ya que mediante el uso de esta dimensión se cuenta con un talento humano eficiente. Cabe recalcar que este tipo de disposición promueve el compromiso entre cada uno de los miembros de la empresa y genera una buena cooperación, de modo que los individuos se unifiquen en la ejecución de sus labores para así lograr las metas comunes.

Así misma una empresa que aplica la estructura horizontal puede mejorar el estilo de vida que tiene su personal, pues tienen una oportunidad de compartir sus responsabilidades, tomar sus propias decisiones y participar en los aspectos más importantes de la organización.

"La especialización horizontal produce aumento de la productividad, debido a que permite el desarrollo de nuevas técnicas, mejora la destreza, ahorra tiempo y facilita la estandarización, pues se permite ubicar a la persona más adecuada en cada puesto" (Arnoletto, 2014). 


\section{La estructura organizacional en función del comportamiento del capital humano en las organizaciones}

Vol. 3, núm. 4., (2019)

Wendy Lorena Ocampo Ulloa; Nora Hursula Huilcapi Masacon; Andrés Fernando Cifuentes Rodríguez

Características de la estructura organizacional horizontal

Una de las claves del éxito que posee aplicar una estructura horizontal se basa en que los niveles operativos de la empresa se unifican para trabajar los unos con otros para así dar paso a cumplir las metas organizacionales, ya que ellos son quienes detectan y dan solución a los inconvenientes que se pueden generar dentro de las actividades que se llevan a cabo en la empresa.

Entre las características que genera la estructura organizativa horizontal, se pueden destacar las siguientes:

- La aplicación de una estructura horizontal facilita la comunicación entre los niveles directivos y el nivel operativo de la entidad.

- Las estructuras horizontales tienden a ser más moldeables, es decir se toman las decisiones de acorde a los aspectos de mayor relevancia lo que da paso a que la empresa ofrezca un buen servicio al cliente. En este sentido es preciso recalcar que el talento humano es quien tiene la potestad de elegir los contextos esenciales para un buen manejo de la organización.

- La organización debe de contar con un personal altamente capacitado, ya que de ellos depende el éxito de la empresa. Por ende deben de desarrollar las habilidades competentes con el fin de asegurar una imagen leal de la entidad y lo más importante que se logre una fidelización con los clientes. 


\section{La estructura organizacional en función del comportamiento del capital humano en las organizaciones}

Vol. 3, núm. 4., (2019)

Wendy Lorena Ocampo Ulloa; Nora Hursula Huilcapi Masacon; Andrés Fernando Cifuentes Rodríguez

Comportamiento del talento humano en función de la estructura horizontal

Es la disciplina que persigue la satisfacción de objetivos organizacionales, para ello es necesario tener una estructura organizativa y la colaboración del esfuerzo humano coordinado. Las organizaciones persiguen objetivos como crecimiento, competitividad, productividad entre otros, mientras que las personas también tienen objetivos individuales: un buen salario, mejorar su calidad de vida, etc.; por ello es importante que las empresas seleccionen a las personas que cumplan los requisitos que las organizaciones desean alcanzar y al mismo tiempo satisfacer las expectativas que las personas desean al ingresar a las organizaciones. (Chávez, 2016, pág. 17).

El talento humano posee libertad, pues pueden tomar sus propias decisiones, mismas que no dependen de la aprobación de alguna autoridad en específico. Una organización que aplica la estructura vertical posee pocos gestores y por ello cada persona trabaja para lograr el bien común de su empresa. En este sentido una de las partes perjudiciales de este tipo de dimensiones es que puede darse la posibilidad de que el talento humano tome decisiones que no sean acertadas y por ende se genere un declive o se puede provocar una mala comunicación entre ciertos empleados lo que distorsione la unificación.

De la estructura vertical a la horizontal

De manera general la estructura organizacional que tiene mayor aplicación es aquella en la cual las actividades están controladas por un nivel directivo o gerencial, pues esta supervisión permite detectar con facilidad como se están llevando a cabo las tareas dentro de la empresa y en 


\section{La estructura organizacional en función del comportamiento del capital humano en las organizaciones}

Vol. 3, núm. 4., (2019)

Wendy Lorena Ocampo Ulloa; Nora Hursula Huilcapi Masacon; Andrés Fernando Cifuentes Rodríguez

caso de existir ciertas desviaciones poder corregirlas a tiempo y así encaminarse hacia el logro de las metas planeadas. De manera específica toda la entidad está coordinada por el gerente, quien es el que toma las decisiones.

Así mismo debido a los avances tecnológicos y a los cambios del entorno pueden darse dificultades en aquellas entidades que aplican una estructura organizacional vertical, ya que los altos mandos no pueden responder con precipitación a las dificultades y oportunidades. En consecuencia es por ello que distintas organizaciones aplican las estructuras organizaciones con carácter horizontal con el fin de que el talento humano sean quienes ejerzan el poder y por ende son los responsables del éxito de su empresa.

En este sentido se puede deducir que es necesario el desarrollo de una estructura organizacional en donde se incluya a todo el talento humano, pues como se mencionó anteriormente los empleados son capacitados con frecuencia para tomar decisiones que contribuyan de manera positiva a la entidad, a su vez que ellos trabajan de manera ardua para satisfacer a sus clientes. Por ende se les debe de reconocer una retribución en compensación del aporte para con la entidad.

La organización debe de valorar los esfuerzos de su talento humano para desarrollar cada una de las actividades que le fueron encomendadas de acorde a la estructura organizacional que la entidad aplica. 


\section{La estructura organizacional en función del comportamiento del capital humano en las organizaciones}

Vol. 3, núm. 4., (2019)

Wendy Lorena Ocampo Ulloa; Nora Hursula Huilcapi Masacon; Andrés Fernando Cifuentes Rodríguez

\section{Resultados.}

Al analizar las respuestas obtenidas de las encuestas aplicadas al personal operativo y directivo de varias empresas de la ciudad de Babahoyo, y denotar la interacción y el comportamiento humano podemos identificar que:

El 75\% de la muestra tomada (4 empresas) dirigidas al nivel directivo utilizan el modelo de estructura organizacional vertical.

Según los resultados obtenidos se demuestra que el $70 \%$ del personal operativo encuestado, conoce el modelo de estructura que aplica la empresa y la importancia de su aporte en beneficio para una transformación favorable para la institución, esto permitirá que exista una interacción activa entre directores departamentales y personal operativo.

Es importante que el talento humano de una empresa conozca el modelo de estructura que se aplica, esto permitirá que exista la correcta fluidez de comunicación entre jefe - empleado, y contar con una institución organizada que tenga óptimo rendimiento.

Preguntas para el nivel gerencial

Tabla 1. Tipo de estructura organizacional

\begin{tabular}{|l|r|r|}
\hline \multicolumn{1}{|c|}{$\begin{array}{c}\text { ¿QUÉ TIPO DE ESTRUCTURA } \\
\text { ORGANIZACIONAL SE APLICA EN SU } \\
\text { EMPRESA? }\end{array}$} & $\begin{array}{r}\text { \# DE } \\
\text { RESPUESTA }\end{array}$ & PORCENTAJE \\
\hline Vertical & 3 & $75 \%$ \\
\hline Horizontal & 1 & $25 \%$ \\
\hline Total & $\mathbf{4}$ & $\mathbf{1 0 0 \%}$ \\
\hline
\end{tabular}




\section{La estructura organizacional en función del comportamiento del capital humano en las organizaciones}

Vol. 3, núm. 4., (2019)

Wendy Lorena Ocampo Ulloa; Nora Hursula Huilcapi Masacon; Andrés Fernando Cifuentes Rodríguez

Discusión: Como se puede observar, el $75 \%$ de los administradores encuestados mencionaron que el tipo de estructura organizacional aplicada en su empresa es vertical y el $25 \%$ de los encuestados dijeron que en su empresa aplican la estructura horizontal.

Tabla 2. Interacción de los gerentes con el nivel operativo

\begin{tabular}{|l|r|r|}
\hline $\begin{array}{c}\text { iDENTRO DE LA ORGANIZACIÓN EL } \\
\text { GERENTE O LOS JEFES DEPARTAMENTALES } \\
\text { INTERACTUNAN CON EL PERSONAL } \\
\text { OPERATIVO? }\end{array}$ & $\begin{array}{c}\text { \# DE } \\
\text { RESPUESTA }\end{array}$ & PORCENTAJE \\
\hline Si & 4 & $100 \%$ \\
\hline No & 0 & $0 \%$ \\
\hline A veces & 0 & $0 \%$ \\
\hline Total & $\mathbf{4}$ & $\mathbf{1 0 0 \%}$ \\
\hline
\end{tabular}

Discusión: Podemos observar que el $100 \%$ de los encuestados respondieron que en la organización interactúan el gerente y jefes departamentales con el personal operativo para el buen funcionamiento de la empresa y cumplir los objetivos.

Tabla 3. Importancia de aplicar una estructura organizacional

\begin{tabular}{|l|r|r|}
\hline $\begin{array}{c}\text { ¿POR QUÉ CONSIDERA UD. QUE ES } \\
\text { IMPORTANTE LA ESTRUCTURA } \\
\text { ORGANIZACIONAL APLICADA EN SU } \\
\text { EMPRESA? }\end{array}$ & \# DE \\
Organización & 1 & PORCENTAJE \\
\hline Mejor Comunicación & 1 & $25 \%$ \\
\hline Rendimiento & 2 & $50 \%$ \\
\hline Total & $\mathbf{4}$ & $\mathbf{1 0 0 \%}$ \\
\hline
\end{tabular}

Discusión: Según los datos arrojados en la encuesta, el 25\% consideran que la organización es importante en la estructura de una empresa, el 25\% mencionaron que la mejor comunicación es importante en la estructura organizacional de la empresa y el 50\% de los encuestados consideran que el rendimiento es importante en la estructura organizacional de la empresa. 


\section{La estructura organizacional en función del comportamiento del capital}

humano en las organizaciones

Vol. 3, núm. 4., (2019)

Wendy Lorena Ocampo Ulloa; Nora Hursula Huilcapi Masacon; Andrés Fernando Cifuentes Rodríguez

Tabla 4. Incidencia de la estructura vertical u horizontal en el comportamiento del talento humano

\begin{tabular}{|l|r|r|}
\hline $\begin{array}{c}\text { ¿CREE UD. QUE EL DESARROLLO DE UNA } \\
\text { ESTRUCTURA VERTICAL U HORIZONTAL EN SU } \\
\text { EMPRESA INCIDE CON EL COMPORTAMIENTO } \\
\text { DE SU TALENTO HUMANO? }\end{array}$ & \#ESPUESTA & PORCENTAJE \\
\hline Si & 4 & $100 \%$ \\
\hline No & 0 & $0 \%$ \\
\hline Total & $\mathbf{4}$ & $\mathbf{1 0 0 \%}$ \\
\hline
\end{tabular}

Discusión: Como se puede observar en el gráfico, el 100\% de los encuestados coinciden que el desarrollo de una estructura vertical y horizontal en su empresa incide con el comportamiento de su talento humano.

Preguntas para el nivel operativo (personal)

Tabla 5. Conocimiento respecto a las estructuras verticales u horizontales

\begin{tabular}{|l|r|r|}
\hline \multicolumn{1}{|c|}{$\begin{array}{c}\text { ¿CONOCE USTED LO QUE ES UNA } \\
\text { ESTRUCTURA ORGANIZACIONAL } \\
\text { VERTICAL Y HORIZONTAL? }\end{array}$} & $\begin{array}{c}\text { \# DE } \\
\text { RESPUESTA }\end{array}$ & PORCENTAJE \\
\hline Si & 7 & $70 \%$ \\
\hline No & 3 & $30 \%$ \\
\hline Total & $\mathbf{1 0}$ & $\mathbf{1 0 0 \%}$ \\
\hline
\end{tabular}

Discusión: Según las encuestas el $30 \%$ no conoce lo que es una estructura organizacional vertical y horizontal, mientras que el $70 \%$ tiene conocimiento lo que es una estructura organizacional vertical y horizontal.

Tabla 6. Aplicación de las estructuras en una empresa

\begin{tabular}{|l|r|r|}
\hline \multicolumn{2}{|c|}{$\begin{array}{c}\text { ¿SABE QUE TIPO DE ESTRUCTURA SE } \\
\text { APLICA EN SU EMPRESA? }\end{array}$} & \# DE \\
& RESPUESTA & PORCENTAJE \\
\hline Si & 7 & $70 \%$ \\
\hline No & 3 & $30 \%$ \\
\hline
\end{tabular}




\section{La estructura organizacional en función del comportamiento del capital}

humano en las organizaciones

Vol. 3, núm. 4., (2019)

Wendy Lorena Ocampo Ulloa; Nora Hursula Huilcapi Masacon; Andrés Fernando Cifuentes Rodríguez

Total

Discusión: En cuanto a los datos arrojados en las encuestas el $30 \%$ no sabe qué tipo de estructura se aplica en su empresa, mientras que $70 \%$ conoce sabe el tipo de estructura de su empresa u organización.

Tabla 7. Participación en ideas transformadoras para beneficio de la organización

\begin{tabular}{|l|r|r|}
\hline $\begin{array}{c}\text { ¿EL PERSONAL PARTICIPA EN IDEAS } \\
\text { TRANSFORMADORAS PARA BENEFICIO DE } \\
\text { LA ORGANIZACIÓN? }\end{array}$ & $\begin{array}{r}\text { \# DE } \\
\text { RESPUESTA }\end{array}$ & PORCENTAJE \\
\hline Si & 5 & $50 \%$ \\
\hline No & 2 & $20 \%$ \\
\hline A veces & 3 & $30 \%$ \\
\hline Total & $\mathbf{1 0}$ & $\mathbf{1 0 0 \%}$ \\
\hline
\end{tabular}

Discusión: Según los datos del gráfico el 20\% no participan en cuanto a ideas transformadoras para beneficio de la organización, el 30\% a veces participa con ideas y el $50 \%$ de los encuestados son participes con ideas para beneficio de la empresa u organización.

Tabla 8. Consideración respecto a si la estructura organizacional es favorable para la institución

\begin{tabular}{|l|r|r|}
\hline $\begin{array}{c}\text { ¿CONSIDERA USTED QUE LA ESTRUCTURA } \\
\text { ORGANIZACIONAL QUE SE APLICA ES } \\
\text { FAVORABLE PARA LA INSTITUCIÓN? }\end{array}$ & $\begin{array}{c}\text { \# DE } \\
\text { RESPUESTA }\end{array}$ & PORCENTAJE \\
\hline Si & 7 & $70 \%$ \\
\hline No & 3 & $30 \%$ \\
\hline Total & $\mathbf{1 0}$ & $\mathbf{1 0 0 \%}$ \\
\hline
\end{tabular}

Discusión: En las encuestas realizadas el 30\% no considera que la estructura organizacional sea favorable para la institución en la que laboran y el 70\% de los encuestados consideran que la estructura organizacional que se aplica en la empresa u organización si es favorable para la organización. 


\section{La estructura organizacional en función del comportamiento del capital humano en las organizaciones}

Vol. 3, núm. 4., (2019)

Wendy Lorena Ocampo Ulloa; Nora Hursula Huilcapi Masacon; Andrés Fernando Cifuentes Rodríguez

\section{Conclusiones.}

Con la información recabada se puede evidenciar que el modelo de estructura organizacional que aplique una empresa es de mucha importancia, así como también que los directivos y el personal operativo conozca de ella, ya que esto permitirá promover una cultura de compromiso y responsabilidad de quienes conforman la institución.

La participación activa de los directivos y su interacción con los colaboradores conlleva a que la organización obtenga un comportamiento organizacional eficaz, permitiendo esto mejoras en el desarrollo y la producción de la empresa.

A pesar que el entorno resiste cambios acelerados y continuos que producen varios efectos en las instituciones y sus estrategias se deben generar mecanismos de responsabilidad de los directivos donde ejerzan su influencia de manera directa o indirecta.

Según las entrevistas realizadas se puede comprobar que la estructura organizacional vertical, es la que se aplica en la mayoría de las empresas encuestadas, misma que se basa en un nivel jerárquico, en la que cuenta con gerentes, jefes departamentales y personal de servicios. Dentro de la cual cada departamento tiene bien definido cuales son las responsabilidades que debe de cumplir, el sistema vertical ha incitado que el personal actué y cumpla de manera adecuada sus labores. 


\section{La estructura organizacional en función del comportamiento del capital humano en las organizaciones}

Vol. 3, núm. 4., (2019)

Wendy Lorena Ocampo Ulloa; Nora Hursula Huilcapi Masacon; Andrés Fernando Cifuentes Rodríguez

\section{Bibliografía.}

Armas Ortega, Y. M., LLanos Enacalada, M. D., \& Traverso Holguin , P. A. (2017). Gestion del talento humano y nuevos escenarios laborales. En Gestion del talento humano y nuevos escenarios laborales (pág. 64). Samborondon, Ecuador.

Arnoletto, E. J. (2014). FUNDAMENTOS DE LA ADMINISTRACION DE ORGANIZACIONES. Cordoba, Argentina: EDUMED. Obtenido de Fundamentos de la administracion de las organizaciones.

Chávez, L. V. (2016). Gestión del Talento Humano. Riobamba, Ecuador: ESPOCH.

Conecionesan. (17 de 03 de 2017). La estructura organizacional funcional. Recuperado el 16 de 07 de 2019, de https://www.esan.edu.pe/apuntes-empresariales/2017/03/la-estructuraorganizacional-funcional/

Enciclipedia Financiera. (27 de 08 de 2014). Estructura Organizativa Horizontal. Recuperado el 15 de 07 de 2019, de https://www.enciclopediafinanciera.com/definicion-estructurahorizontal.html

Gonzales, A. (11 de 01 de 2018). Emprende Pyme. Recuperado el 15 de 07 de 2019, de https://www.emprendepyme.net/organigrama-vertical.html

Llanos Encalada, M., Pacheco Rodríguez, M. G., Romero Vélez, E. M., Coello Arrata, F., \& Armas Ortega, Y. M. (2016). LA CULTURA ORGANIZACIONAL- EJE DE ACCIÓN DE LA GESTIÓN HUMANA. (M. Á. Laucirica, Ed.) Samborondón, Ecuador.

Logan, E. (01 de 02 de 2018). Cuidatudinero. Recuperado el 17 de 07 de 2019, de https://www.cuidatudinero.com/13105197/las-diferencias-entre-las-estructurasorganizativas-verticales-y-horizontales

Martínez, C. A. (23 de 04 de 2014). Gestiopolis. Recuperado el 17 de 07 de 2019, de https://www.gestiopolis.com/tipos-de-organizacion-y-estructura-organizacional/

Mejias, A. (18 de Marzo de 2019). Cuida tu dinero. Recuperado el 15 de 07 de 2019, de https://www.cuidatudinero.com/13117251/la-estructura-organizativa-vertical-y-lahorizontal

Mejias, A. (18 de 03 de 2019). Cuida tu dinero. Recuperado el 15 de 07 de 2019, de https://www.cuidatudinero.com/13117251/la-estructura-organizativa-vertical-y-lahorizontal

Mora, M. d. (2014). Estructura del area de Gestion del talento humano. Tesis de Grado, 4. Cuenca, Ecuador . 


\section{La estructura organizacional en función del comportamiento del capital} humano en las organizaciones

Vol. 3, núm. 4., (2019)

Wendy Lorena Ocampo Ulloa; Nora Hursula Huilcapi Masacon; Andrés Fernando Cifuentes Rodríguez

Pazmiño Romero, D., Caicedo Monserrate, D., \& Ocampo Ulloa, W. (15 de 02 de 2018). Artiiculo Cientidico, Vol. 2 núm, 15. Ecuador : Saberes del Conocimiento.

Stephen , R., \& Judge, T. (2013). Comportamiento Organizacional (13 Edicion ed.). (F. H. Carrasco, Ed.) Mexico: Pearson.

$$
\text { (9) }(1)(0)
$$

RECONOCIMIENTO-NOCOMERCIAL-COMPARTIRIGUAL

CC BY-NC-SA

ESTA LICENCIA PERMITE A OTROS ENTREMEZCLAR, AJUSTAR Y CONSTRUIR A PARTIR DE SU OBRA CON FINES NO COMERCIALES, SIEMPRE Y CUANDO LE RECONOZCAN LA AUTORÍA Y SUS NUEVAS CREACIONES ESTÉN BAJO UNA LICENCIA CON LOS MISMOS TÉRMINOS. 